\title{
V. ERRA'TA
}

Errata au Bulletin geodésique, 1937.

Bulletin $\mathrm{n}^{\prime 36}$. Th. Niethammer : Die Auswahl der Sterne in der Bestimmung der Zeit und des Azimutes mit Hilfe von Meridiandurchgängen.

1. $27^{5}$, ligne 2. Au lieu de (1), lire (2).

281 , 3. Supprimer als $90^{\circ}$.

282, "19. Au lieu de den, lire der.

283, "6. Formule (1o):

au numérateur, an liea de $\cos \Phi$, lire $\sin \Phi$, au dénominateur, - $\sin \Phi,-\cos \Phi$.

284. " 6 (du texte). Au lieu de es, lire er.

289. " 8. Au lieu de sol, lire soll.

\section{Errata au Bulletin géodésique, 1938.}

Bulletin $n^{\circ} 57$. H.W. Dahling: Notes on the compulation of elliptic integrals in some special cases.

P. 13, ligne $10,2^{\circ}$ colonne. ،la lieu de from log sin $\beta^{3}$, lire from log $\sin \beta_{3}$.

17, ligne 2. Au lieu de increased, lire decheased.

W. D. LAMBER'T and F. W. DaRling: Formulas and Tables for the deflection of the vertical.

P. $5_{1}$, ligne 3 , équation $\left(b_{2}\right)$, à la fin de la ligne (fin du premier membre de l'équation). Au lieu de $d$, lire $d \theta$. 


\section{Errata}

aux Tables de l'ellipsoïde de référence international adopté à l'Assemblée générale de Madrid en 1924.

(Publications spéciales $\mathrm{n}^{\text {0 }}{ }_{2}$ et 3

du Secrétariat de l'Association internationale de Géodésie de l'Union géodésique et géophysique internationale ').

Corrections communes aux Tables dans le système sexagésimal (Publication spéciale $\mathbf{n}^{\circ}$ 2) el aux Tables dans le système centésimal (Publication spéciale $\mathbf{n}^{\circ} 3$ ).

Préface, même pagination pour les deux Tables :

$\begin{array}{rlrl}\text { Page 14: } & \text { Au lieu de : } & \text { lire }: \\ \log (a-b)= & 4 \cdot 331954484706 \quad & \ldots \ldots \ldots \ldots \ldots & 4708 \\ \log n & =\overline{3} \cdot 2369 \$ 5306685 & \ldots \ldots \ldots \ldots \ldots & 6636\end{array}$

Page 17: Alu lieu de : lire :

$$
\mathrm{N}_{\mathrm{c}}=\frac{a \sqrt{\mathrm{I}-e^{2}}}{\mathrm{I}^{2}} \quad \mathrm{~N}_{\mathrm{i}}=\frac{c}{\mathrm{~T}^{2}} \quad \sqrt{\mathrm{N}_{\mathrm{i}}}=\frac{a \sqrt{\mathrm{I}-e^{2}}}{\mathrm{~W}^{2}} \quad \sqrt{\mathrm{N}_{\mathrm{\beta}}}=\frac{c}{\mathrm{~V}^{2}}
$$

Correclions aux Tables dans le système sexagésimal (Publication spéciale $n^{* 2}$ ).

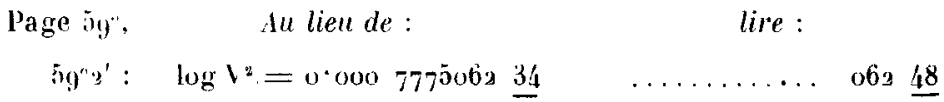

Page tín".

$64^{\circ} 9^{\prime}: \quad \log V^{2}=0.000563146033 \quad \ldots \ldots \ldots \ldots .46083$

3. Corrections signalées le 7 mars 1939 par le Dr V. K. Ölander, de l'Institut géodésique de Finlande à Helsinki, que nous remercions vivement ici. - G. P. 